\title{
A Study of Relationship between Body Mass Index and the Onset of Menarche
}

\author{
Dr. Rupam Ajeet $\operatorname{Yadav}^{1}$, Jyoti Bala Choubey ${ }^{2}$ \\ Assistant Professor, Department of H.Sc, Bhilai Mahila Mahavidyalaya \\ Assistant Professor, Department of H.Sc, Bhilai Mahila Mahavidyalaya
}

\begin{abstract}
Adolescence is a period of transition from girl to womanhood and puberty is a normal phenomenon of Adolescence. The landmarks of pubertal events in girls are onset of puberty, increase in height and the onset of menarche.Age of Menarche has an important health implication on girls because early age of Menarche is associated with several adverse health effects like eating disorders, depression. Type-2 Diabetes in adulthood, breast cancer, coronary Heart disease and overall mortality. Studies indicate that mean age of Menarche has decreased in last 40 years, due to increased incidences of obesity. Reduced physical activity, availability of health facilities and consumption of fast food are associated with increased BMI i.e. overweight and obesity. The purpose of this study is to find out whether higher BMI values are associated with early age of menarche. A cross sectional study was conducted on 50 randomly selected girls between 10-16 years of age. Results indicate that subjects with high BMI values attained menarche earlier than underweight or normal weight subjects.
\end{abstract}

Keywords: Puberty, menarche

\section{Introduction}

Adolescence is a period of transition from girl to womanhood and puberty is a normal phenomenon of Adolescence. During this period progress of secondary sexual characteristics lead to total differentiation between male and female.

The landmarks of pubertal events in girls are onset of puberty, increase in height and the onset of menarche. Menarche indicates female fertility uptomenopause. Mohammad K. et.al.(2005). Age of Menarche has an important health implication on girls because early age of Menarche is associated with several adverse health effects like eating disorders, depression. C Kaltial et.al. (2003) Type-2 Diabetes in adulthood, breast cancer, coronaryHeart disease and overall mortality. It also leads to 'Loss of childhood' among young girls.

There are several factors responsible for early age of Menarche, such as Heredity, typeof diet, lifestyle factors and socioeconomic status etc. Of the various factors childhood obesity is the prime factor. Ahmed et.al \&Walvoor.

Studies indicate that mean age of Menarche has decreased in last 40 years, due to increased incidences of obesity. Kaplowitz (2006). Reduced physical activity, availability of health facilities and consumption of fast food are associated with increased BMI i.e. overweight and obesity.

Early menarche causes premature closure of epiphyseal plates, thus women with early age of menarche have a shorter final height than other women. Earlier puberty is associated with psychological problem, breast cancer and also polycystic ovary syndrome. Chronic malnutrition delays the onset of puberty.
In Industrialized Nations, the age of menarche has decreased to a large extent due to improvement in Nutrition, Sanitation and general health.

Obesity epidemic is one of the important factors in the decline of age of puberty. The purpose of this study is to find out whether higher BMI values are associated with early age of menarche. According to Mumby et. al. 2011 early menarche is considered before the age of 12. For starting of menstrual cycle $17 \%$ of body fat is required. Increase in body fat percent leads to earlier menarche.

\section{Methodology}

A cross sectional study was conducted on 50 randomly selected girls between 10-16 years of age from families living in different sectors of Bhilai township. Information regarding date of menarche, physical activity and diet was collected using a questionnaire after taking their consent.

\section{Result \&Discussion}

Results indicate that subjects with high BMI values attained age of menarche earlier than underweight or normal weight subjects.

In underweight category $91.3 \%$ of girls attained menarche in normal age. In normal weight category $98.2 \%$ girls attained menarche in normal age but in overweight and obese category only $38.5 \%$ had normal age of menarche and $61.5 \%$ had earlier menarche. 


\section{International Journal of Science and Research (IJSR) \\ ISSN (Online): 2319-7064 \\ Index Copernicus Value (2016): 79.57 | Impact Factor (2015): 6.391}

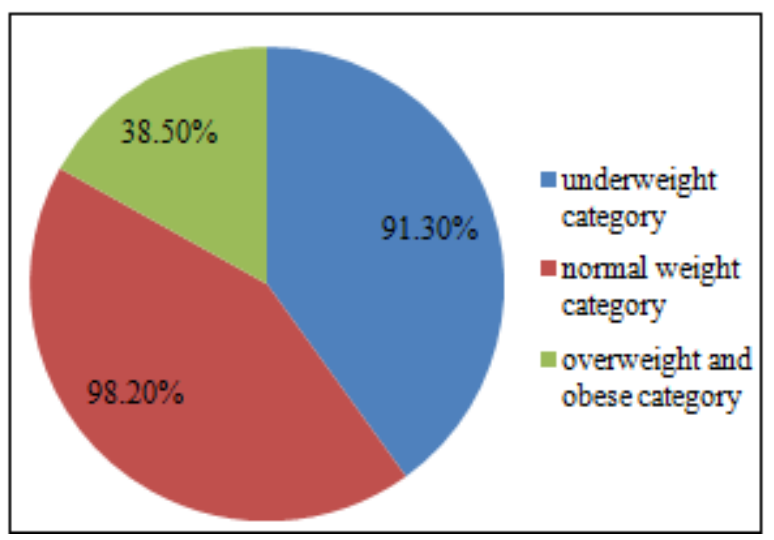

The study denotes that diet and pattern of exercise must be monitored by parents and children to maintain more natural rate of physical development Exercise maintains neuroendocrine system which allows normal physical development children are spending more time watching TV and playing video games, eating fast food and calorie dense foods. Burkey et.al. (2000)

High intake of animal protein is associated with earlier onset of menarche because it causes obesity and prematurity. According to Mumby et.al. 2011. For every $1 \mathrm{~kg} / \mathrm{m} 2$ increase in childhood BMI, there was a $65 \%$ increase in possibility of attaining early Menarche.
Table 1: Relationship between Body Mass Index and Onset

\begin{tabular}{|c|c|c|c|c|}
\hline \multicolumn{5}{c}{ of Menarche } \\
\hline BMI Category & Onset & Early & Normal & Total \\
\hline \multirow{3}{*}{ Underweight } & Frequency & 02 & 21 & 23 \\
\cline { 2 - 5 } & Row \% & $8.7 \%$ & $91.3 \%$ & $100 \%$ \\
\cline { 2 - 5 } & Column \% & $16.7 \%$ & $15.2 \%$ & $15.3 \%$ \\
\hline \multirow{3}{*}{ Normal } & Frequency & 02 & 112 & 114 \\
\cline { 2 - 5 } & Row \% & $1.8 \%$ & $98.2 \%$ & $100 \%$ \\
\cline { 2 - 5 } & Column \% & $16.7 \%$ & $81.2 \%$ & $76 \%$ \\
\hline \multirow{3}{*}{ Overweight and obese } & Frequency & 02 & 112 & 114 \\
\cline { 2 - 5 } & Row \% & $61.5 \%$ & $38.5 \%$ & $100 \%$ \\
\cline { 2 - 5 } & Column \% & $66.7 \%$ & $3.6 \%$ & $76 \%$ \\
\hline Total & Count & $\mathbf{1 2}$ & $\mathbf{1 3 8}$ & $\mathbf{1 5 0}$ \\
\hline
\end{tabular}

\section{$x 2(\mathrm{df}=2)=56.68, \mathrm{p}<0.01$}

Pearson $r=0.344, p<0.01$

Perusal of entries reported in table 1 shows that $8.7 \%$ girls from under weight category had early onset of menarche, $1.8 \%$ girls from normal weight category had early onset of menarche while $61.5 \%$ obese and overweight girls had early onset of menarche. The calculated value $\times 2(\mathrm{df}=2)=56.68$ and Pearson correlation coefficient of 0.344 are statistically significant at 0.01 level. On the basis of results it can be interpreted that there is a significant relationship between BMI and onset of menarche.

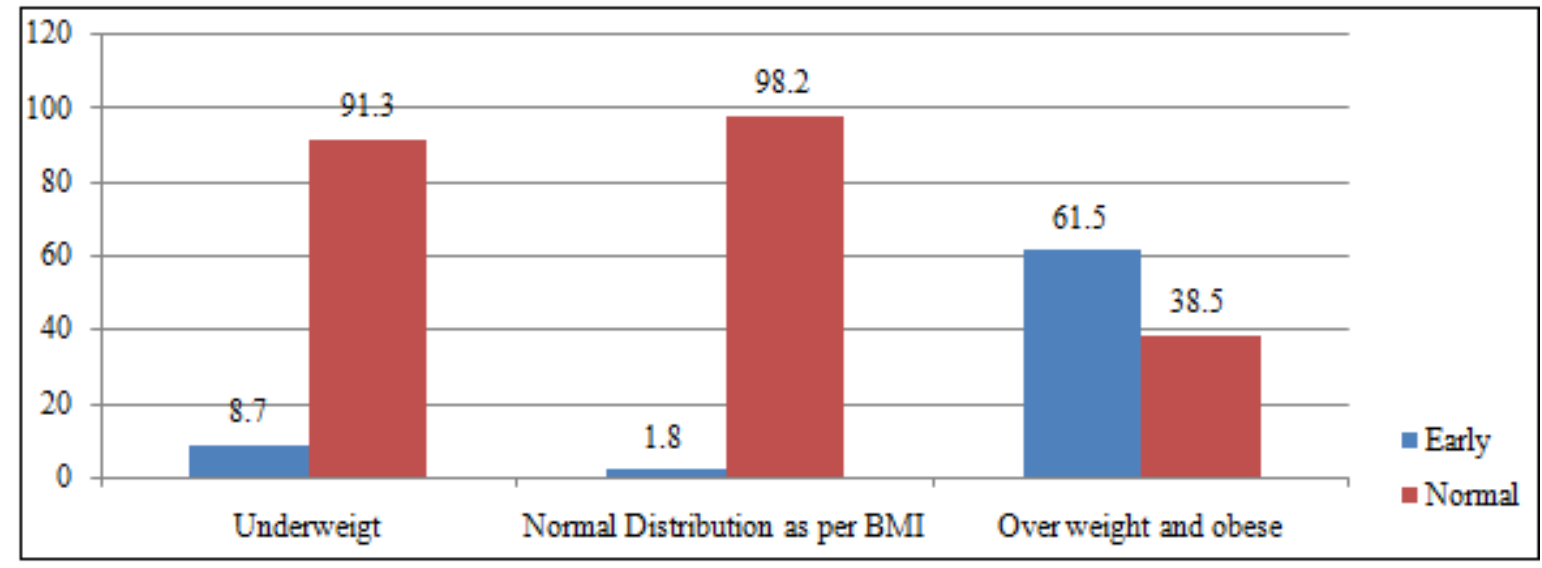

Figure 1: Bar Diagram Showing Relationship between BMI and Onset of Menarche

\section{Result}

A relationship was observed between Body Mass Index and Onset of menarche, Obese and overweight girls have markedly higher tendency to attain menarche earlier than the normal age,as compared to girls with normal weight and under weight category.

\section{References}

[1] Mohammad K, Zerati H, Madzadeh R, Korimloo M (2005) To investigate the Menarcheal age means of Iranian girls. Iranian Journal 4:523-30

[2] Kaltiala, H. R., Kosuner E, Rimpela M, Pubertal timing, sexual behavior and sex reported depression in middle adolescence J Adolescence - 2003; 26(5):531-545.

[3] Lakshman R, Forouhi N, Luben R, Birigham S, Khawk Wareham N, Ong K. K, (2008) Association between age of menarche and risk of Diabetes in Adults Diabetologia $51(50: 781-786$

[4] Kaplowitz P (2006) Pubertal development in girls CurrOpinObstelGynecol 18(5): 487-91

[5] Ahmed ML, Ong KK, Dunger DB (2009) childhood obesity and the timing of puberty TEM 2009, 20(5) : 237-242

[6] WalvoordEC(2010) The timing of puberty Is it changing ? does it matter? The Journal of adolescent health 47(5): 433-439

[7] Berkey C.S., Garner J. D., Frazier AC and colditz GA (2000) Relation to childhood diet and body size to menarche and adolescent growth in girls Am. J. epidemiol 152:446-452. 